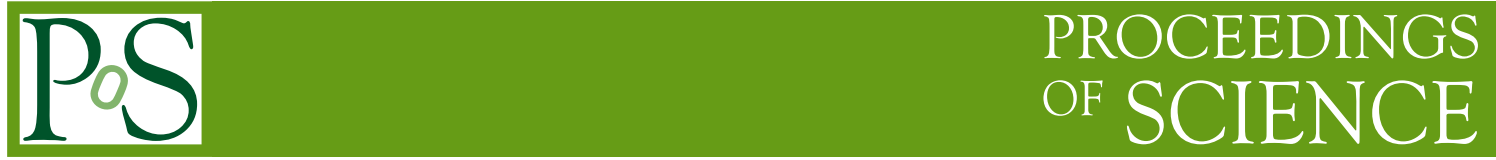

\title{
Mixing kaons with mixed action chiral perturbation theory
}

\section{Aubin*}

Physics Department, Columbia University, New York, NY 10027, USA

E-mail: caubin@phys. columbia.edu

\section{Jack Laiho and Ruth S. Van de Water}

Theoretical Physics Department, Fermilab, Batavia, IL 60510, USA

We calculate the neutral kaon mixing parameter, $B_{K}$, to next-to-leading order in mixed action (domain-wall valence with staggered sea quarks) chiral perturbation theory. We find the expression for $B_{K}$ in this mixed-action case only differs from the continuum partially quenched expression by an additional analytic term. Additionally, in preparation for a lattice calculation of $B_{K}$ with a mixed action, we discuss quantitatively the effects of the taste violations as well as finite volume effects.

Tucson, Arizona, USA

\footnotetext{
${ }^{*}$ Speaker.
} 
The kaon $B$-parameter, $B_{K}$, parameterizes the hadronic contribution to $K^{0}-\bar{K}^{0}$ mixing. It is crucial in extracting information about the CKM matrix using experimental measurements of the neutral kaon system. The dominant source of error in constraining the CKM matrix using $K^{0}-\bar{K}^{0}$ mixing currently comes from the lattice determination of $B_{K}$.

Current calculations of $B_{K}$ use either domain-wall quarks [1,2] or improved staggered quarks $[3,4]$, both of which have their advantages and disadvantages. For domain-wall quarks, the presence of good chiral symmetry significantly reduces the effects of operator mixing and thus makes the renormalization procedure simpler, but the numerical cost currently makes it difficult to reach very light quark masses. For staggered quarks, the case is the opposite: One can reach rather light quark masses (down to $\approx m_{s} / 10$ ), yet the appearance of wrong-taste operators makes renormalization complicated. Additionally, the use of the "fourth-root trick" is not without controversy due to taste violations at finite $a$. Although the issue regarding the validity of this "fourth-root trick" is far from resolved $[6,7,8,9,10]$, there are strong indications that this technique is valid, and as such we assume its validity in this work. For more details on the fourth-root trick, refer to Sharpe's plenary talk [5] and references therein. There have been numerous results using staggered quarks that show excellent agreement with experimental results $[11,12,13]$.

To make use of the advantages of both techniques while bypassing the disadvantages, one can adopt a "mixed action" approach by using valence domain-wall quarks on top of a staggered sea. By using staggered sea quarks and domain-wall valence quarks one can better approach the chiral regime in the sea sector while minimizing operator mixing and allowing the use of nonperturbative renormalization. Since the MILC staggered lattices with $2+1$ flavors of dynamical quarks are publicly available and offer a number of quark masses and lattice spacings [14, 15], mixed-action simulations can be performed at the same cost as quenched domain-wall simulations. Mixed-action simulations have already been successfully used to study quantities of interest to nuclear physics (see, for example, Refs. [16, 17]); we expect that a similar method can be used to calculate the weak matrix element $B_{K}$.

We present here a summary of our results for calculating $B_{K}$ in mixed-action chiral perturbation theory $(\chi \mathrm{PT})$ with domain-wall valence and staggered sea quarks (a complete description of the calculation of $B_{K}$ with this approach can be found in Ref. [19]). The results we present here are applicable to simulations with purely Ginsparg-Wilson $(\mathrm{G}-\mathrm{W})$ valence quarks with staggered sea quarks, however a replacement of $m^{\mathrm{val}} \rightarrow m^{\mathrm{val}}+m_{\text {res }}$, with $m_{\text {res }}$ the residual mass, will make our results relevant for domain-wall quarks with a staggered sea.

The theoretical basis for mixed-action $\chi \mathrm{PT}$ was first presented in Ref. [18]. We consider a partially quenched theory with $2 \mathrm{G}-\mathrm{W}$ valence quarks $(x, y)$ and 3 staggered sea quarks $(u, d$, and $s) .{ }^{1}$ Each staggered sea quark comes in four tastes, and each G-W valence quark has a corresponding bosonic ghost partner to cancel its contribution to loop diagrams. The mixed action theory has an approximate $S U(14 \mid 2)_{L} \otimes S U(14 \mid 2)_{R}$ graded chiral symmetry which becomes exact in the combined massless quark and continuum limit. In analogy with QCD, we assume that chiral symmetry spontaneously breaks to its vector subgroup,

$$
S U(14 \mid 2)_{L} \otimes S U(14 \mid 2)_{R} \rightarrow S U(14 \mid 2)_{V}
$$

\footnotetext{
${ }^{1}$ In Ref. [19], the more general case of an arbitrary number of sea and valence quarks is discussed.
} 
and gives rise to 255 pions. ${ }^{2}$ These pions can be packaged into the nonlinear field $\Sigma=\exp (2 i \Phi / f)$, where $\Phi$ contains the pions and $f$ is normalized such that $f_{\pi} \approx 131 \mathrm{MeV}$. Under chiral symmetry transformations, $\Sigma \rightarrow L \Sigma R^{\dagger}$, with $L, R \in S U(14 \mid 2)_{L, R}$. We use the standard power-counting scheme: $\varepsilon \sim p^{2} \sim m_{q} \sim a^{2}$, so the lowest-order, $\mathscr{O}(\varepsilon)$, mixed action chiral Lagrangian is

$$
\mathscr{L}=\frac{f^{2}}{8} \operatorname{Str}\left(\partial_{\mu} \Sigma \partial_{\mu} \Sigma^{\dagger}\right)-\frac{\mu f^{2}}{4} \operatorname{Str}\left(\Sigma M^{\dagger}+M \Sigma^{\dagger}\right)+a^{2}\left(\mathscr{U}_{S}+\mathscr{U}_{S}^{\prime}+\mathscr{U}_{V}\right)
$$

where Str indicates a graded supertrace over both flavor and taste indices and $\mu$ is an undetermined dimensionful parameter. $\mathscr{U}_{S}$ and $\mathscr{U}_{S}^{\prime}$ comprise the well-known staggered potential and come from taste-symmetry breaking in the sea quark sector [20], and $\mathscr{U}_{V}$ is a taste-symmetry breaking term which mixes the sea and valence sectors [18]. Except for the contributions from $\mathscr{U}_{S}$ to the tree-level mass splittings for the staggered mesons, we will not need these three terms in the Lagrangian.

The leading-order expression for the masses of the mesons are

$$
\left(m_{x y}^{\mathrm{LO}}\right)^{2}=\mu\left(m_{x}+m_{y}\right), \quad\left(m_{f f^{\prime}, t}^{\mathrm{LO}}\right)^{2}=\mu\left(m_{f}+m_{f^{\prime}}\right)+a^{2} \Delta_{t}^{f f^{\prime}}
$$

where $f, f^{\prime}$ are both sea quarks, and $\Delta_{t}^{f f^{\prime}}$ depends on the taste $t \in\{P, V, A, T, I\}$ of the meson (with $\left.\Delta_{P}^{f f^{\prime}}=0\right)$. Pions with one sea and one valence quark do not appear in this calculation.

The spurion analysis for $B_{K}$ in the mixed action case is similar to that in the continuum [21,22]. Recall that $\left\langle\bar{K}^{0}\left|\mathscr{O}_{K}\right| K^{0}\right\rangle=\frac{8}{3} m_{K}^{2} f_{K}^{2} B_{K}$, with, at the quark level

$$
\mathscr{O}_{K}=\left[\bar{y} \gamma_{\mu}\left(1-\gamma_{5}\right) x\right]\left[\bar{y} \gamma_{\mu}\left(1-\gamma_{5}\right) x\right]
$$

At leading order, we find that the chiral operator has precisely the same form as the continuum [19]

$$
\mathscr{O}_{K}^{\chi}=\frac{2}{3} B_{0} f^{4} \sum_{\mu} \operatorname{Str}\left[\Sigma \partial_{\mu} \Sigma^{\dagger} P_{\bar{y} x}\right] \operatorname{Str}\left[\Sigma \partial_{\mu} \Sigma^{\dagger} P_{\bar{y} x}\right]
$$

with $P_{\bar{y} x}$ projecting out a valence kaon and $B_{0}$ an unknown parameter (the value of $B_{K}$ at tree level). This operator is of order $\mathscr{O}\left(p^{2}\right)$, and so we must include possible terms of $\mathscr{O}\left(m_{q}\right)$ or $\mathscr{O}\left(a^{2}\right)$. Terms involving the mass are not allowed as they would not give rise to chiral operators transforming correctly under the symmetry group. There could in principle be terms that violate the taste symmetry and thus arise at $\mathscr{O}\left(a^{2}\right)$, but a simple analysis shows that these do not appear at this order in the chiral expansion [19]. Thus, $\mathscr{O}_{K}^{\chi}$ is the only operator which gives rise to one-loop non-analytic terms for $B_{K}$.

Next-to-leading order analytic contributions to $B_{K}$ come from tree-level matrix elements of NLO, or $\mathscr{O}\left(\varepsilon^{2}\right)$, operators. There are many such operators in the mixed-action chiral Lagrangian which we will not enumerate here, since it is not necessary to separate them in fits to numerical lattice data. Using symmetry arguments, we can restrict the form of the analytic terms. Following Ref. [22], we find there are only four possible terms that can appear:

$$
\left(\frac{B_{K}}{B_{0}}\right)^{\text {analytic }}=c_{1} a^{2} m_{x y}^{2}+c_{2} m_{x y}^{4}+c_{3}\left(m_{X}^{2}-m_{Y}^{2}\right)^{2}+c_{4} m_{x y}^{2}\left(m_{U_{P}}^{2}+m_{D_{P}}^{2}+m_{S_{P}}^{2}\right) .
$$

\footnotetext{
${ }^{2}$ We use the term "pion" to refer to any of the pseudo-Goldstone bosons.
} 
The details of the calculation as well as the most general results for $B_{K}$ can be found in Ref. [19]. Here we will just show the expression for $2+1$ partially quenched theory in which $m_{u}=m_{d} \neq m_{s}$ in the sea sector. Above are the relevant analytic terms, and the 1-loop expression is

$$
\begin{aligned}
\left(\frac{B_{K}}{B_{0}}\right)^{\text {loop }}= & +\frac{1}{16 \pi^{2} f_{x y}^{2} m_{x y}^{2}}\left[-2 m_{x y}^{4} \ell^{\prime}\left(m_{x y}^{2}\right)-\ell\left(m_{X}^{2}\right)\left(m_{X}^{2}+m_{x y}^{2}\right)-\ell\left(m_{Y}^{2}\right)\left(m_{Y}^{2}+m_{x y}^{2}\right)\right. \\
& \left.+\frac{1}{3}\left(m_{X}^{2}-m_{Y}^{2}\right)^{2} \frac{\partial}{\partial m_{X}^{2}} \frac{\partial}{\partial m_{Y}^{2}}\left\{\sum_{j} \ell\left(m_{j}^{2}\right)\left(m_{x y}^{2}+m_{j}^{2}\right) R_{j}^{[3,2]}\left(\left\{M_{X Y, I}^{[3]}\right\} ;\left\{\mu_{I}^{[2]}\right\}\right)\right\}\right],
\end{aligned}
$$

with

$$
\left\{M_{X Y, I}^{[3]}\right\} \equiv\left\{m_{X}, m_{Y}, m_{\eta_{I}}\right\}, \quad\left\{\mu_{I}^{[2]}\right\} \equiv\left\{m_{D_{I}}, m_{S_{I}}\right\} .
$$

The chiral logarithms in a finite volume [23] are defined as

$$
\ell\left(m^{2}\right)=m^{2}\left(\ln \frac{m^{2}}{\Lambda_{\chi}^{2}}+\delta_{1}^{F V}(m \mathrm{~L})\right), \quad \delta_{1}^{F V}(m \mathrm{~L})=\frac{4}{m \mathrm{~L}} \sum_{\vec{r} \neq 0} \frac{K_{1}(|\vec{r}| m \mathrm{~L})}{|\vec{r}|},
$$

where the difference between the finite and infinite volume result is given by $\delta_{1}^{F V}(m \mathrm{~L})$, and $K_{1}$ is a modified Bessel function of imaginary argument. For the infinite volume result, one sets $\delta_{1}=0$. In Eq. (8) the prime indicates derivative with respect to the argument. Finally, the residues in Eq. (7) are defined to be

$$
R_{j}^{[n, k]}(\{m\},\{\mu\}) \equiv \frac{\prod_{a=1}^{k}\left(\mu_{a}^{2}-m_{j}^{2}\right)}{\prod_{i \neq j}\left(m_{i}^{2}-m_{j}^{2}\right)} .
$$

The definition of the "full QCD" point, where the valence and sea pions have the same mass, is ambiguous in the mixed theory since there are multiple pions in the sea sector one could match the valence masses to. For the subsequent numerical discussion, we match the pseudoscalar taste sea pion to the valence pion, and for simplicity we scale the valence quark masses so that they are numerically the same as the sea quark masses at the full QCD point. This is to say that while the input valence light mass is $m_{0}^{\mathrm{val}}$, we plot our results as a function of $m_{l}^{\mathrm{val}}=\frac{Z_{m}^{\mathrm{DW}}}{Z_{m}^{\text {stag }}}\left(m_{0}^{\mathrm{val}}+m_{\mathrm{res}}\right)$ for ease of comparison. One merely needs to invert this expression to determine what the input domain-wall mass would be in this case.

The NLO expression for $B_{K}$ in a mixed action theory with $2+1$ flavors of sea quarks is given in Eq. (7). Discretization errors lead to two contributions - the shift in the mass-squared of the taste-singlet sea-sea meson that appears in the 1-loop disconnected contribution, and the analytic term proportional to $a^{2}$. The new mixed-action parameter (coming from $\mathscr{U}_{V}$ in the mixed-action Lagrangian) does not arise in the NLO expression for $B_{K}$. Since the taste-singlet splitting has already been measured on the MILC lattices [15], this leaves only one unknown parameter. We stress, however, that this parameter does not only come from taste violations, and a similar term would appear in a purely domain-wall expression.

For our numerical analysis, we will neglect $c_{1}$, since we do not know the value of this $\mathscr{O}\left(a^{2}\right)$ term. We choose to study discretization errors using the parameters on the $a \approx 0.125 \mathrm{fm}$ "coarse" MILC lattices since taste violations will be more pronounced than on the finer lattices. In particular, we use the parameters of the ensemble with the lightest up and down sea quark masses on the 
smaller volume $(L / a=20)$; this ensemble has a light quark mass of $a m_{l}^{\text {sea }}=0.007$ and a strange quark mass of $a m_{s}^{\text {sea }}=0.05$.

In order to estimate the size of discretization errors in $B_{K}$, we calculate the percent difference between the 1-loop contributions to $B_{K}$ with and without taste-breaking:

$$
\eta=\frac{B_{K}^{1-\text { loop }}\left(m_{l}^{\mathrm{val}}, a^{2} \Delta_{I}\right)-B_{K}^{1-\mathrm{loop}}\left(m_{l}^{\mathrm{val}}, 0\right)}{B_{K}^{1-\text { loop }}\left(m_{l}^{\mathrm{val}}, 0\right)} .
$$

In this expression we have set the heavier valence bare quark mass to be equal to the sea strange bare quark mass so that $\eta$ is a function of the light valence quark mass, $m_{l}^{\text {val }}$, and the taste-singlet splitting, $a^{2} \Delta_{I}$. The taste singlet meson is the heaviest of all of the staggered sea-sea mesons, and $a^{2} \Delta_{I}$ is approximately $(450 \mathrm{MeV})^{2}$ on the coarse lattices. Because the only sea-sea mesons that contribute to the $B_{K}$ at 1-loop in the mixed action theory are taste-singlets, this large splitting makes the effective sea quark mass considerably larger than a nominal light sea quark mass of $m_{s} / 10$ or $m_{s} / 7$ would suggest. On the fine lattices this splitting is much less, by a factor of 9 , so it is necessary to approach the continuum limit in order to approach the chiral limit in the sea sector.

Fig. 1(a) shows the percent difference, Eq. (11), as a function of valence quark mass, with $a^{2} \Delta_{I}$ set to be the value measured in MILC simulations on the coarse lattices [15]. In this plot the star is the full QCD point defined by setting the valence and sea quark masses to be equal (as discussed above), while the vertical line shows the location of the physical value of the average up/down quark mass, $m_{l}^{\text {phys }} \approx m_{s} / 27$. For larger valence light masses, $\eta$ rapidly vanishes as expected, since the difference between the masses of the valence and sea mesons will ultimately be neglible for sufficiently large quark masses. At quark masses near or below the full QCD point, $\eta$ begins to diverge rapidly as $m_{l}^{\text {val }} \rightarrow 0$. Note, however, that this does not begin to happen until below the physical mass, so in the region of interest the error coming from taste violations is never higher than $0.5 \%$.

We now repeat the above analysis, focussing on errors due to the finite size of the lattice. Such finite volume effects can be quite noticeable at the lightest sea quark masses available on the MILC configurations. Since partially quenched pathologies begin to appear when $m_{\pi}^{\mathrm{val}}<m_{\pi}^{\mathrm{sea}}$, and the relevant sea mass is that of the (rather large) taste-singlet pion mass, these finite volume effects are larger than one would naively expect as one decreases the valence mass.

In analogy with Eq. (11), we define $\eta_{F V}$ to be the percent difference between the 1-loop contribution to $B_{K}$ in the mixed theory at finite volume and $B_{K}$ in the mixed theory at infinite volume, both including discretization errors:

$$
\eta_{F V}\left(m_{l}^{\mathrm{val}}, a^{2} \Delta_{I}\right)=\frac{B_{K}^{1-\mathrm{loop}, \mathrm{FV}}\left(m_{l}^{\mathrm{val}}, a^{2} \Delta_{I}, L\right)-B_{K}^{1-\mathrm{loop}}\left(m_{l}^{\mathrm{val}}, a^{2} \Delta_{I}\right)}{B_{K}^{1-\mathrm{loop}}\left(m_{l}^{\mathrm{val}}, a^{2} \Delta_{I}\right)} .
$$

We evaluate the above expression at a spatial lattice size of $L=20$; the remaining parameters are the same as in the previous analyisis. In Fig. 1(b) we show two curves - the dashed curve shows the percent difference in Eq. (12) for the continuum limit, $\eta_{F V}\left(m_{l}^{\text {val }}, 0\right)$, while the solid curve shows the same percent difference with $a^{2} \Delta_{I}$ set to its value on the coarse MILC lattices. Again, the star corresponds to the full QCD point and the vertical line indicates the physical light quark mass.

One can see that, for the full QCD point and larger masses, the error associated with finite volume effects, while not negligible, is quite small, of $\mathscr{O}(1 \%)$ or less. It is when we drop below the 


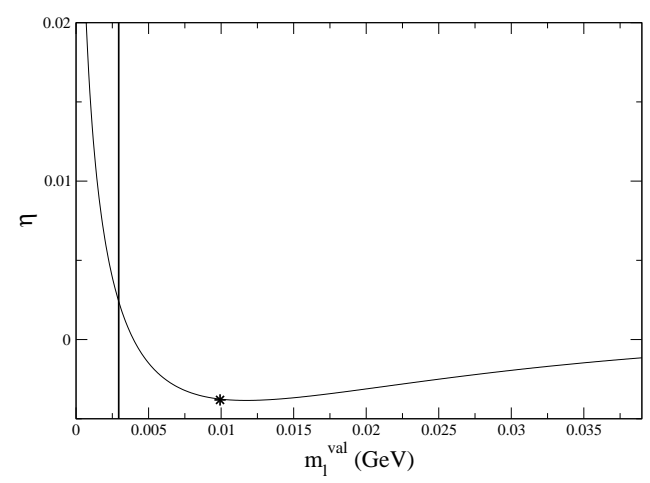

(a)

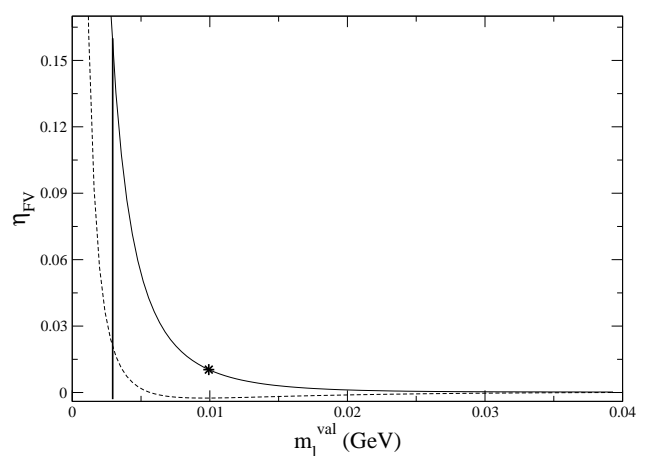

(b)

Figure 1: (a) $\eta$ as a function of valence light mass. (b) $\eta_{F V}$ as a function of valence light mass. The dashed line is for $\Delta_{I}=0$ and the solid line is with this splitting set to the value on the MILC coarse lattices.

full QCD point where the difference between the continuum and finite $a$ cases becomes rather large. The error for the finite $a$ case becomes unreasonably large almost immediately upon decreasing the valence mass below the full QCD point, up to $15 \%$ at the physical point. This could be a source of worry theoretically, but practically this is not a problem. In actual simulations, as the masses are lowered, the volume eventually increases, so as to always stay to the right of the "wall" in Fig. 1(b). For example, the MILC ensemble with a light sea quark mass of $a m_{l}^{\text {sea }}=0.005$ has a spatial length of $L=24$ as opposed to 20 at the heavier masses. Thus, as long as one remains in the region where the errors are small (near or above the full QCD point in the plot), they can easily be accounted for and should not introduce large systematic errors.

In this work we have calculated the expression for $B_{K}$ in a mixed action lattice theory with Ginsparg-Wilson valence quarks and staggered sea quarks to next-to-leading order in chiral perturbation theory. We have discussed in some detail how to extend the continuum calculation to the mixed action case, and we have provided expressions for a $2+1$ partially quenched theory $\left(m_{u}=m_{d} \neq m_{s}\right.$ ), both of which reduce to the corresponding partially quenched QCD expressions in the continuum limit. We have also performed a numerical analysis to study the taste-violating and finite volume effects and found that the errors for both of these effects are small enough to be under control in a numerical simulation. This calculation shows that a lattice calculation of $B_{K}$ using a mixed action approach is rather similar to a purely domain-wall approach, and as in the domain-wall case, the expression for $B_{K}$ in chiral perturbation theory is strikingly similar to the continuum.

\section{References}

[1] S. D. Cohen, Preliminary study of $B_{K}$ on $2+1$ flavor DWF lattices from QCDOC, PoS LAT2005 (2005) 346, [hep-lat/0602020].

[2] S. D. Cohen. these proceedings.

[3] HPQCD Collaboration, E. Gamiz et al., Unquenched determination of the kaon parameter $B_{K}$ from improved staggered fermions, hep-lat/0603023. 
[4] J. Kim, T. Bae, and W. Lee, Calculating $B_{K}$ using a mixed action, PoS LAT2005 (2005) 338, [hep-lat/0510007].

[5] S. R. Sharpe. these proceedings.

[6] M. Creutz, Flavor extrapolations and staggered fermions, hep-lat / 0603020.

[7] C. Bernard, M. Golterman, Y. Shamir, and S. R. Sharpe, Comment on 'Flavor extrapolations and staggered fermions', hep-lat/0603027.

[8] C. Bernard, M. Golterman, and Y. Shamir, Observations on staggered fermions at non-zero lattice spacing, hep-lat/0604017.

[9] Y. Shamir, Renormalization-group analysis of the validity of staggered-fermion qcd with the fourth-root recipe, hep-lat/0607007.

[10] C. Bernard, Staggered chiral perturbation theory and the fourth-root trick, Phys. Rev. D73 (2006) 114503, [hep-lat/0603011].

[11] HPQCD, Fermilab, MILC, and UKQCD Collaborations, C. T. H. Davies et al., High-precision lattice QCD confronts experiment, Phys. Rev. Lett. 92 (2004) 022001, [hep-lat / 0304004 ].

[12] MILC Collaboration, C. Aubin et al., Light pseudoscalar decay constants, quark masses, and low energy constants from three-flavor lattice QCD, Phys. Rev. D70 (2004) 114501, [hep-lat/0407028].

[13] HPQCD Collaboration, I. F. Allison et al., Mass of the $B_{c}$ meson in three-flavor lattice QCD, Phys. Rev. Lett. 94 (2005) 172001, [hep-lat/ 0411027 ].

[14] C. W. Bernard et al., The QCD spectrum with three quark flavors, Phys. Rev. D64 (2001) 054506, [hep-lat/0104002].

[15] C. Aubin et al., Light hadrons with improved staggered quarks: Approaching the continuum limit, Phys. Rev. D70 (2004) 094505, [hep-lat / 0402030 ].

[16] Lattice Hadron Physics Collaboration, B. Bistrovic et al., Understanding hadron structure from lattice QCD in the SciDAC era, J. Phys. Conf. Ser. 16 (2005) 150-159.

[17] NPLQCD Collaboration, S. R. Beane, P. F. Bedaque, K. Orginos, and M. J. Savage, I = 2 pi pi scattering from fully-dynamical mixed-action lattice QCD, Phys. Rev. D73 (2006) 054503, [hep-lat/0506013].

[18] O. Bär, C. Bernard, G. Rupak, and N. Shoresh, Chiral perturbation theory for staggered sea quarks and Ginsparg-Wilson valence quarks, Phys. Rev. D72 (2005) 054502, [hep-lat / 0503009 ].

[19] C. Aubin, J. Laiho, and R. S. Van de Water, The kaon b-parameter in mixed action chiral perturbation theory, hep-lat/0609009.

[20] C. Aubin and C. Bernard, Pion and kaon masses in staggered chiral perturbation theory, Phys. Rev. D68 (2003) 034014, [hep-lat/0304014].

[21] J. Bijnens, H. Sonoda, and M. B. Wise, On the validity of chiral perturbation theory for $K^{0}$ anti- $K^{0}$ mixing, Phys. Rev. Lett. 53 (1984) 2367.

[22] R. S. Van de Water and S. R. Sharpe, $B_{K}$ in staggered chiral perturbation theory, hep-lat/0507012.

[23] MILC Collaboration, C. Bernard, Chiral logs in the presence of staggered flavor symmetry breaking, Phys. Rev. D65 (2002) 054031, [hep-lat/ 0111051 ]. 